\title{
Pericytes limit tumor cell metastasis
}

\author{
Xiaojie Xian, ${ }^{1}$ Joakim Håkansson, ${ }^{2}$ Anders Ståhlberg, ${ }^{1}$ Per Lindblom, ${ }^{3}$ \\ Christer Betsholtz, ${ }^{4}$ Holger Gerhardt, ${ }^{3}$ and Henrik Semb ${ }^{1}$
}

\begin{abstract}
${ }^{1}$ Stem Cell Center, Lund University, Lund, Sweden. ${ }^{2}$ Department of Medical Biochemistry, Sahlgrenska Academy at Göteborg University, Gothenburg, Sweden. ${ }^{3}$ Vascular Biology Laboratory, Cancer Research UK, London, United Kingdom. ${ }^{4}$ Laboratory of Vascular Biology, Division of Matrix Biology, Department of Medical Biochemistry and Biophysics, and Department of Medicine, Karolinska Institutet, Stockholm, Sweden.
\end{abstract}

\begin{abstract}
Previously we observed that neural cell adhesion molecule (NCAM) deficiency in $\beta$ tumor cells facilitates metastasis into distant organs and local lymph nodes. Here, we show that NCAM-deficient $\beta$ cell tumors grew leaky blood vessels with perturbed pericyte-endothelial cell-cell interactions and deficient perivascular deposition of ECM components. Conversely, tumor cell expression of NCAM in a fibrosarcoma model (T241) improved pericyte recruitment and increased perivascular deposition of ECM molecules. Together, these findings suggest that NCAM may limit tumor cell metastasis by stabilizing the microvessel wall. To directly address whether pericyte dysfunction increases the metastatic potential of solid tumors, we studied $\beta$ cell tumorigenesis in primary pericyte-deficient $P d g f b^{\text {ret } / \text { ret }}$ mice. This resulted in $\beta$ tumor cell metastases in distant organs and local lymph nodes, demonstrating a role for pericytes in limiting tumor cell metastasis. These data support a new model for how tumor cells trigger metastasis by perturbing pericyte-endothelial cell-cell interactions.
\end{abstract}

\section{Introduction}

Metastasis is the principal cause of cancer-treatment failure and death in cancer patients. Metastasis may occur through different routes, including lymphatic and hematogenous spreading, local tissue invasion, and direct seeding of body cavities or surfaces (1). Whereas tumor cell spreading as a consequence of local invasion has been shown to involve changes in cell-cell adhesion, cell-ECM adhesion, cell motility, and epithelial-mesenchymal conversion of tumor cells (2), the underlying cause for the escape of tumor cells through the blood vasculature is largely unknown.

By using a multistage pancreatic $\beta$ cell tumor model, Rip1Tag2 (RT) (3), we recently demonstrated that neural cell adhesion molecule (NCAM) regulates metastatic tumor cell dissemination independently of the invasive properties of the tumor cells. Whereas RT tumors do not metastasize, approximately $50 \%$ of RT mice lacking 1 or 2 functional NCAM alleles developed metastases to distant organs and local lymph nodes, indicating both hematogenous and lymphatic spreading of the tumor cells. Reexpression of NCAM-120 specifically in $\beta$ tumor cells prevented metastasis, demonstrating that the causal role of NCAM in limiting tumor cell spreading takes place within $\beta$ tumor cells and not within the host stroma (4). Importantly, NCAM expression undergoes significant changes in human cancer. In colon carcinoma, pancreatic cancer, and astrocytoma, NCAM expression is markedly downregulated, which correlates with poor prognosis (5-7). However, the underlying mechanism for NCAM's role in tumor progression, including metastasis, has not been clarified.

In the process of angiogenesis, newly formed blood vessels become stabilized through recruitment of vascular mural cells (VSMCs or pericytes) and by the formation of a perivascular ECM including the vascular basement membrane. Pericytes, the mural cells of microvessels, extend long cytoplasmic processes on the abluminal surface of the endothelial cells, making tight contacts that are important for blood vessel stabilization,

Nonstandard abbreviations used: NCAM, neural cell adhesion molecule; PDGFR, PDGF receptor; RT, Rip1Tag2.

Conflict of interest: The authors have declared that no conflict of interest exists. Citation for this article: J. Clin. Invest. doi:10.1172/JCI25705. remodeling, and function (8-10). During both developmental and tumor angiogenesis, the recruitment of pericytes is regulated by endothelial PDGF-B, which stimulates its receptor, PDGFR- $\beta$, on pericytes (11-15). However, whereas in developmental situations appropriate numbers of pericytes end up in tight association with the abluminal surface of the endothelium, the pericytes surrounding tumor vessels commonly are less abundant and develop abnormal phenotypes, including aberrant cell shape, changes in marker expression, and loose vessel attachment $(9-11,16)$. It is possible that mural cell deficiency contributes to some of the abnormal functional properties of tumor vessels, e.g., increased vessel leakiness. Here, we studied the mechanism of NCAM's role in limiting tumor cell metastasis and asked whether it could be mediated by an effect on tumor vessel pericyte recruitment. By using 2 independent tumor models, we show that tumor cell NCAM promotes integration of pericytes in the vessel wall. Furthermore, the metastatic potential of solid tumors was increased in a genetic mouse model of PDGF-B deficiency and perturbed pericyte-endothelial cell-cell interactions, suggesting that pericytes play a causal role in limiting tumor cell metastasis. It was recently suggested that the increased lymphatic metastasis in NCAM-deficient RT may be linked to an increased expression of lymphangiogenic growth factors, and increased lymphangiogenesis (17). Here we provide evidence for the alternative or complementary scenario that tumor cell NCAM limits $\beta$ tumor cell metastasis through its promotion of pericyte-endothelial cell-cell interactions.

\section{Results}

NCAM-deficient RT tumor progression is associated with increased blood vessel leakage. In agreement with previous analysis of NCAM's role in $\beta$ tumor cell dissemination, all phenotypes reported herein were qualitatively indistinguishable between $\mathrm{RT}^{\mathrm{NCAM+}+-}$ and $\mathrm{RT}^{\mathrm{NCAM-/-}}$ mice (4). Consequently, $\mathrm{RT}^{\mathrm{NCAM+}+-}$ and $\mathrm{RT}^{\mathrm{NCAM}-/-}$ mice are collectively referred to as NCAM-deficient $\mathrm{RT}$ or $\mathrm{RT}^{\mathrm{NC} / \mathrm{KO}}$ mice. Bloodfilled cavities arising as a consequence of extravasations have previously been described in RT tumors $(16,18)$. Histopathological analyses of angiogenic islets ( 8 weeks) demonstrated that the number of islets containing blood-filled cavities in $\mathrm{RT} \mathrm{T}^{\mathrm{NC} / \mathrm{KO}}$ mice 

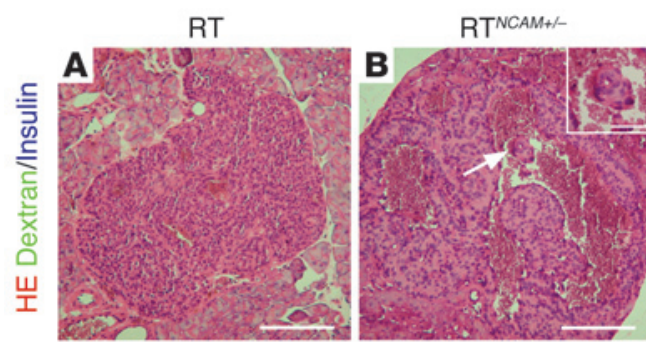

$\mathrm{RT}^{\mathrm{NCAM+1-}}$
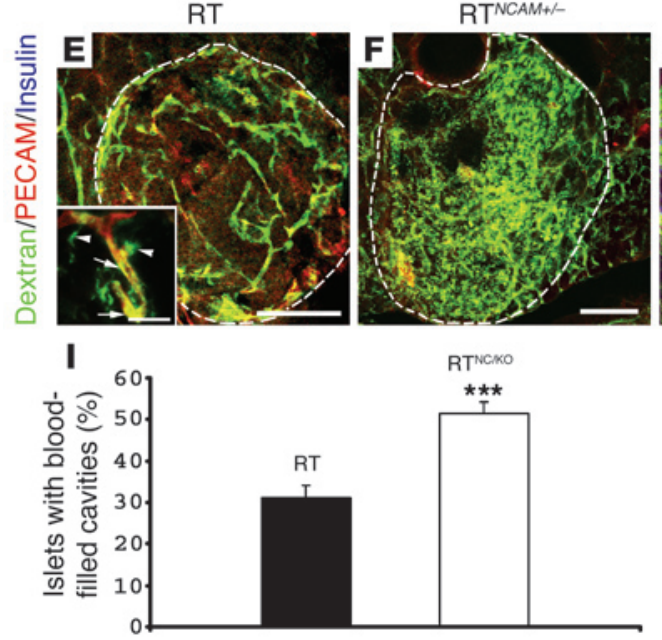
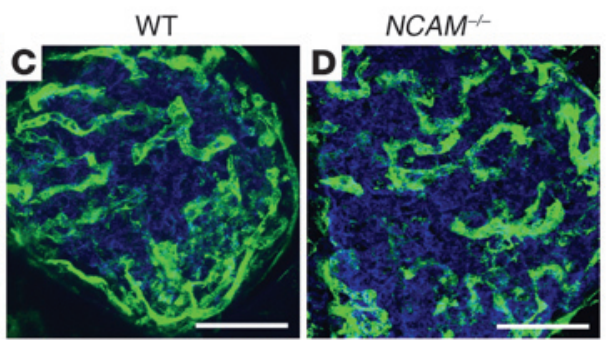

$\mathrm{RT}^{\mathrm{NCAM+1}}$

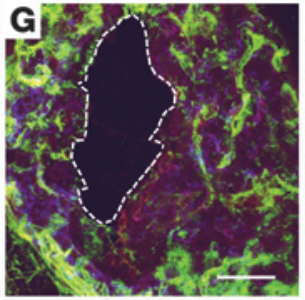

J

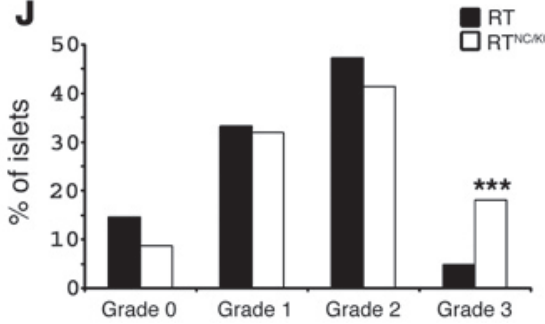

Figure 1

NCAM deficiency induces increased tumor blood vessel leakage during $\beta$ tumor cell progression. Pan-

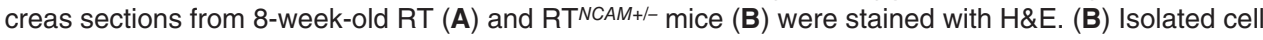
clusters were specifically found inside blood-filled cavities within RTNC/KO islets (arrow). Inset in B shows a higher magnification of the isolated cell cluster. (C-H) Pancreas sections of mice perfused with FITC-dextran (green) were double-immunostained with antibodies against PECAM (red) and insulin (blue). (C and D) Islets from WT (C) and NCAM ${ }^{-1-}$ (D) mice. (E-H) Angiogenic islets from 8-week-old RT (E and G) and $\mathrm{RT}^{N C A M+/-}(\mathbf{F}$ and $\mathbf{H})$ mice. The islet area is indicated by dashed lines, and extravascular and intravascular FITC-dextran is indicated by arrowheads and arrows, respectively, in inset in $\mathbf{E}$. Dashed lines in $\mathbf{G}$ and $\mathbf{H}$ mark blood-filled cavities. FITC-dextran specifically leaked into RTNC/KO blood-filled cavities (H). (I) The percentage of islets containing blood-filled cavities was higher in $\mathrm{RT}^{\mathrm{NC} / K O}(n=292)$ compared with RT $(n=313)$ mice at 8 weeks of age $\left(\chi^{2}\right.$ test, $\left.{ }^{* \star *} P<0.001\right)$. Average values \pm SEM are shown. $(\mathbf{J})$ Distribution of RT $(n=144)$ and RTNC/KO $(n=149)$ islets at 8 weeks of age according to their vessel leakage (grades 0-3, where grade 3 includes islets with most extensive leakage). The percentage of grade 3 islets was significantly higher in RTNC/KO compared with RT mice $\left(\chi^{2}\right.$ test, $\left.{ }^{* * *} P<0.001\right)$. Scale bars: $200 \mu \mathrm{m}$ (A and B), $50 \mu \mathrm{m}(\mathbf{C}, \mathbf{D}, \mathbf{G}, \mathbf{H}$, and inset in B), $100 \mu \mathrm{m}$ (E and $\mathbf{F}$ ), and $25 \mu \mathrm{m}$ (inset in $\mathbf{E}$ ).

was increased by $66 \%$ compared with that in RT mice (Figure 1, A, B, and I). Importantly, isolated tumor cell clusters were only found within $\mathrm{RT}^{\mathrm{NC} / \mathrm{KO}}$ blood-filled cavities (Figure 1B).

To investigate whether the increased number of blood-filled cavities in $\mathrm{RT}^{\mathrm{NC} / \mathrm{KO}}$ islets was associated with increased vessel leakage, we perfused the vasculature with FITC-conjugated dextran. In normal pancreatic islets, blood vessel leakage was insignificant, and NCAM deficiency resulted in no change in leakage (Figure 1, C and D). RT islets demonstrated FITC-dextran leakage in both WT and NCAM-deficient mice, but the percentage of islets with most vessel leakage (grade $3 ;>30 \%$ of islet area covered with FITC-dextran) was significantly higher in $\mathrm{RT} \mathrm{NC}^{\mathrm{NCO}}$ compared with RT mice (Figure 1, E-H and J). Notably, the most extreme leakage ( $>50 \%$ of islet area; Figure $1 \mathrm{~F}$ ) was only observed in $\mathrm{RT}^{\mathrm{NC} / \mathrm{KO}}$ mice ( 9 of 25 grade $3 \mathrm{RT} \mathrm{T}^{\mathrm{NC} / \mathrm{KO}}$ islets compared with 0 of 7 grade $3 \mathrm{RT}$ islets). In further support of more extensive leakage in $\mathrm{RT}^{\mathrm{NC} / \mathrm{KO}}$ islets, we observed leakage of FITC-dextran in 15 of 88 examined RT ${ }^{\mathrm{NC} / \mathrm{KO}}$ intra-islet bloodthe expression patterns of these markers was a higher number of desmin-positive perivascular cells compared with $\alpha$-SMA ${ }^{+}$perivascular cells within nontumorigenic islets (18). For convenience, we used $\alpha$-SMA as a marker for pericytes, but because this marker may to some degree also be expressed by reactive fibroblasts, we refer to cells expressing this marker as $\alpha-\mathrm{SMA}^{+}$cells.

In normal islets, $\alpha-\mathrm{SMA}^{+}$cells were tightly associated with endothelial cells independently of the presence or absence of NCAM (Figure 2, A and B). During tumor progression, however, $\alpha-\mathrm{SMA}^{+}$cells were regularly detached from the endothelium, acquired fibroblast-like cell shapes, and organized into sheetlike structures (Figure 2, C-E). Although these $\alpha-\mathrm{SMA}^{+}$cell phenotypes were indistinguishable between $\mathrm{RT}$ and $\mathrm{RT}^{\mathrm{NC} / \mathrm{KO}}$ tumors at late stages of tumor progression (12-14 weeks of age; data not shown), abnormal $\alpha-\mathrm{SMA}^{+}$cell distribution was more commonly observed in $\mathrm{RT}^{\mathrm{NC} / \mathrm{KO}}$ islets compared with $\mathrm{RT}$ islets during early stages of tumor progression. More specifically, we found a 

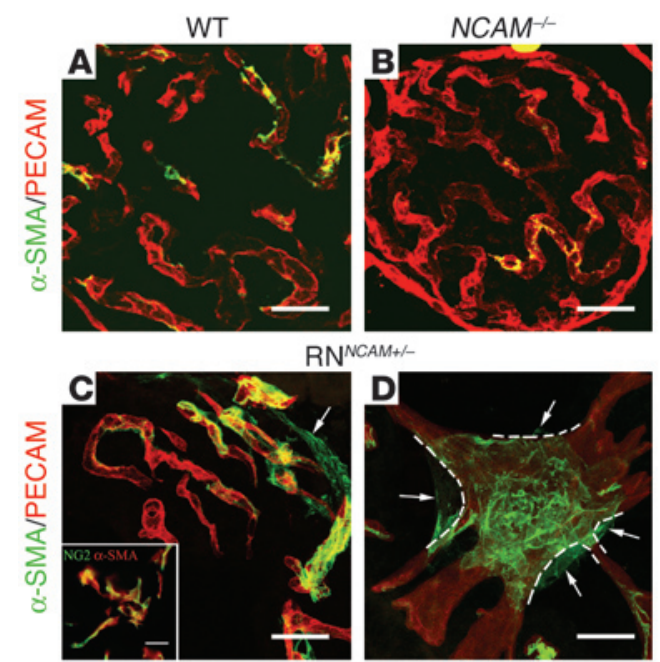

$\alpha-$ SMA/PECAM

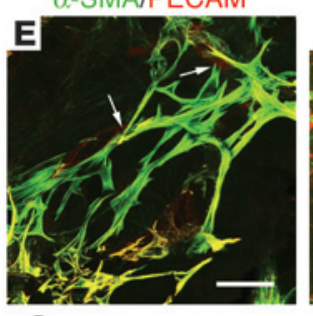

Dextran/ $\alpha-S M A$
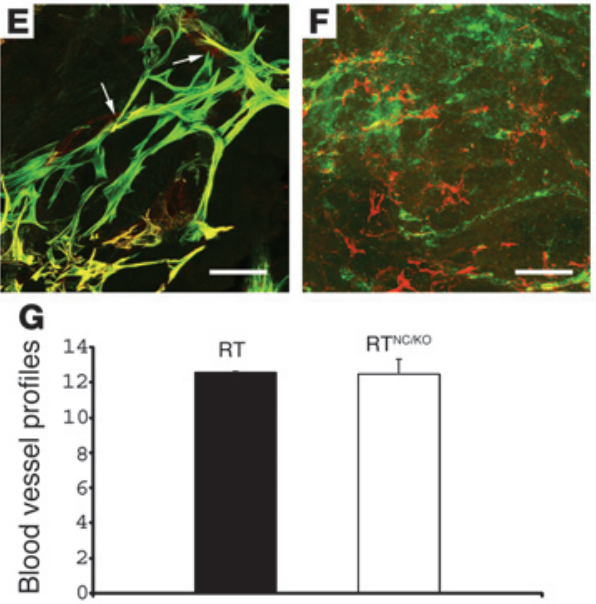

$64 \%$ increase in islets with disturbed mural cell organization in $\mathrm{RT}^{\mathrm{NC} / \mathrm{KO}}$ mice compared with RT mice at 8 weeks of age. Moreover, at 8 weeks of age, the fibroblast-like $\alpha$-SMA ${ }^{+}$cells, which also expressed NG2, were only observed in $\mathrm{RT}^{\mathrm{NC} / \mathrm{KO}}$ angiogenic islets (Figure 2E). These findings suggest that NCAM deficiency results in earlier onset of tumor blood vessel abnormalities, such as dissociation of $\alpha-\mathrm{SMA}^{+} \mathrm{NG}^{+}$cells from the endothelium. These abnormalities did not correlate with changes in vessel density, which was unaffected by NCAM deficiency (Figure 2G). Notably, severe blood vessel leakage (grade 3) correlated spatially with pericyte abnormalities, including vessel-detached $\alpha$-SMA ${ }^{+}$cells arranged in sheet-like structures (Figure $2 \mathrm{~F}$ ), suggesting that the 2 phenomena may be causally related.

To address whether NCAM deficiency generally affects blood vessel development and mural cell function also in normal developmental angiogenesis, we studied endothelial sprouting and vessel and pericyte densities in developing retinas of intercrosses between NCAM-deficient and XlacZ4 mice (the latter of which express lacZ in pericytes) (20). No changes were observed in these parameters at 5 days and 3 weeks after birth, respectively (data not shown). Finally, to investigate whether the vascular phenotypes in the pancreas could be due to general vascular defects in $\mathrm{RT}^{\mathrm{NC} / \mathrm{KO}}$ mice, we analyzed the brain and skin of $\mathrm{RT}^{\mathrm{NC} / \mathrm{KO}}$ mice but found no signs of vascular abnormality at these sites (data not shown). Thus, we

\section{Figure 2}

Pathological organization of periendothelial $\alpha-\mathrm{SMA}^{+}$cells correlates with increased tumor vessel leakage in RTNC/KO angiogenic islets. Pancreas sections from 8-week-old mice were double-immunostained with antibodies against PECAM (red) and $\alpha-S M A(\mathbf{A}-\mathbf{E}$, green; $\mathbf{F}$, red). In WT $(\mathbf{A})$ and $\mathrm{NCAM}^{-/-}(\mathrm{B})$ islets, $\alpha-\mathrm{SMA}^{+}$cells were closely attached to the endothelium. Premature abnormal organization of periendothelial $\alpha-\mathrm{SMA}^{+}$cells, including detachment of $\alpha-\mathrm{SMA}^{+}$cells from endothelial cells (arrow in C) and multiple layers of $\alpha-\mathrm{SMA}^{+}$cells with an apparent loose attachment to the endothelium (D), and presence of fibroblastlike $\alpha-\mathrm{SMA}^{+}$cells in RTNCAM+/- angiogenic islets (E), were observed in $\mathrm{RT}^{\mathrm{NCAM+}+\text { - }}$ islets. The dashed lines and arrows in $\mathbf{D}$ indicate the borders of the endothelium and $\alpha-\mathrm{SMA}^{+}$cells stretching away from the vessel, respectively. (F) Pancreas section of an 8-week-old RT ${ }^{N C A M+/-}$ mouse perfused with FITC-dextran (green), immunostained with anti- $\alpha$-SMA (red). Increased leakage correlated with severely disorganized $\alpha-S M A^{+}$ periendothelial cells. The inset in $\mathbf{C}$ shows coexpression of NG2 (green) and $\alpha$-SMA (red). (G) Analysis of blood vessel density revealed no difference between $R T$ and $R T^{N C / K O}$ islets. Average values \pm SEM are shown. Scale bars: $50 \mu \mathrm{m}(\mathbf{A}-\mathbf{F})$.

conclude that lack of either 1 or 2 NCAM alleles affects blood vessel integrity in RT tumor angiogenesis but does not significantly disturb normal developmental angiogenesis.

NCAM promotes pericyte recruitment during tumor angiogenesis. To address whether NCAM plays a general role in tumor angiogenesis, we studied its influence on tumor progression in a skin fibrosarcoma tumor model (T241). Transplantation of T241 tumor cells results in encapsulated fibrosarcomas that develop all the hallmarks of blood vessel changes associated with tumor angiogenesis, i.e., deficient coverage of the endothelium by pericytes and aberrant blood vessel morphology (11). The fact that T241 cells do not normally express NCAM allowed us to test whether NCAM plays a general role in limiting vascular dysfunction during tumor angiogenesis by a gain-of-function approach. Interestingly, expression of NCAM in T241 cells (T241 $\left.{ }^{\mathrm{NCAM}}\right)$ increased pericyte recruitment (47\%) and coverage (33\%) (Figure 3, A-C), strengthening the idea that NCAM normally promotes interactions between perivascular and endothelial cells. Moreover, expression of NCAM resulted in increased tumor vessel density (27\%) (Figure 3D) and increased tumor size (2-fold) in comparison with untransfected and enhanced GFP-transfected (eGFP-transfected) T241 (T241 ${ }^{\text {eGFP }) ~ c e l l s ~(F i g u r e ~ 3, ~ E ~ a n d ~ F) . ~ N C A M ~ a n d ~ e G F P ~ e x p r e s-~}$ sion was confirmed with immunohistochemistry, and measurements of growth rates showed that the NCAM-mediated increase in tumor growth in vivo was not due to an increase in cell proliferation in vitro (data not shown). Thus, these results suggest that NCAM is sufficient to prevent vascular dysfunction during tumorigenesis by promoting cell interactions between periendothelial and endothelial cells. To investigate whether the increase in tumor mass in vivo was caused by increased cell proliferation or diminished apoptosis or both, we quantified Ki67 and caspase- 3 by immunofluorescence. More $\mathrm{Ki} 67^{+}$and caspase- $3^{+}$cells were detected in T241 ${ }^{\mathrm{NCAM}}$ tumors, indicating an increase in both cell proliferation and apoptosis compared with $\mathrm{T} 241^{\mathrm{eGFP}}$ tumors.

To address whether the blood vessel phenotypes involved homophilic interactions between NCAM molecules on tumor and host cells, e.g., pericytes or stromal cells, we transplanted T241 ${ }^{\mathrm{eGFP}}$ and T241 ${ }^{\text {NCAM }}$ cells on NCAM-deficient mice. However, no change in the vascular phenotype and tumor cell growth was observed compared with when these cells were transplanted on WT mice (Figure 3F 
A
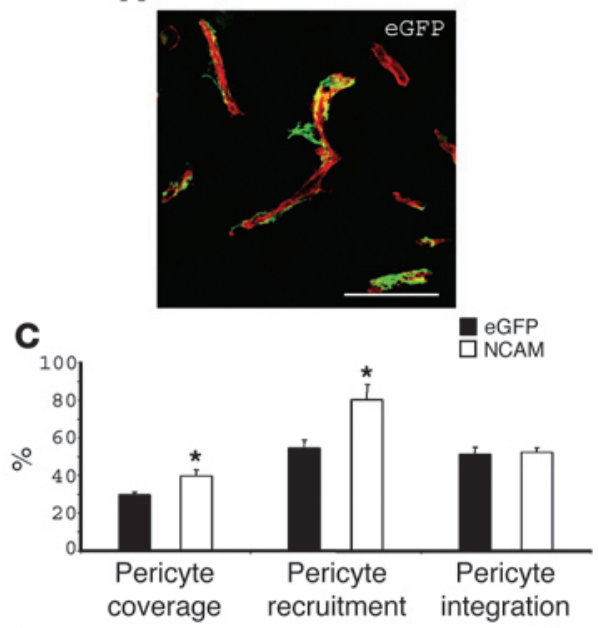

$\mathbf{E}$

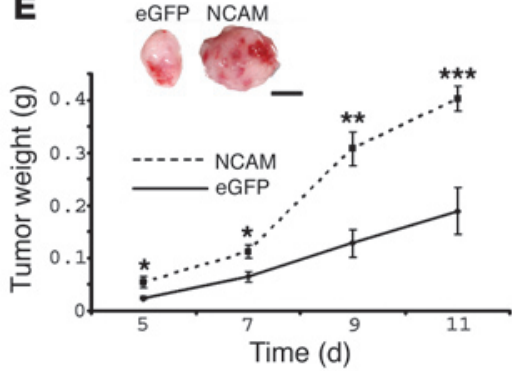

B
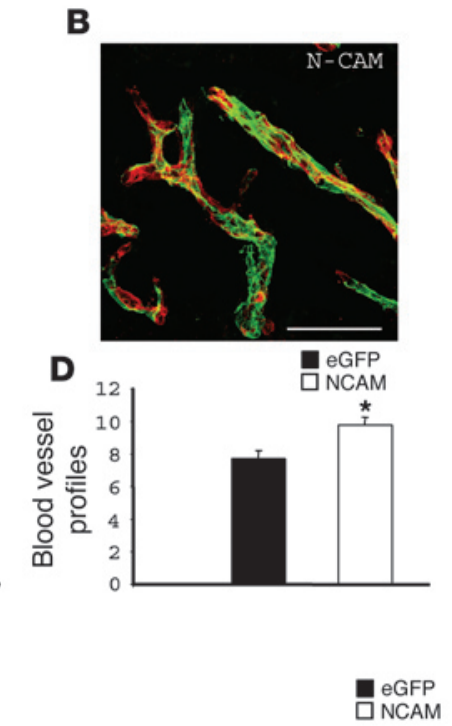

$\mathbf{F}$

\section{Figure 3}

Ectopic expression of NCAM in T241 tumor cells stimulates tumor growth and improves pericyte recruitment and coverage. (A and $\mathbf{B}$ ) Tumor sections of T241 eGFP (A) and T241NCAM (B) cells transplanted on WT mice double-stained with antibodies against PECAM (red) and $\alpha$-SMA (green). (C and D) Pericyte recruitment and coverage, but not integration (C), together with the number of vessel profiles (D), increased in T241 NCAM compared with T241 eGFP tumors. (E and F) Independent of whether grafted on WT (E and F) or NCAM-/- $(\mathbf{F})$ mice, growth of T241 NCAM tumors (WT, $n=17$; $\mathrm{NCAM}^{-/-}, n=10$ ) was significantly increased compared with growth of T241 ${ }^{\text {GGFP }}$ tumors (WT, $n=20$; CAM $^{-/-}, n=10$ ) (F). (E) Tumor growth curves of T241 $\mathrm{eGFP}$ and T241 NCAM tumor cells grafted on WT mice ( $n=6-8$ for each genotype and time point). The inset shows examples of tumors at day 9. Average values \pm SEM are shown. Scale bars: $50 \mu \mathrm{m}(\mathbf{A}$ and $\mathbf{B})$ and $5 \mathrm{~mm}(\mathbf{E}) .{ }^{*} P<0.05$, ${ }^{\star *} P<0.01,{ }^{* * *} P<0.001$ (2-tailed Student's $t$ test).

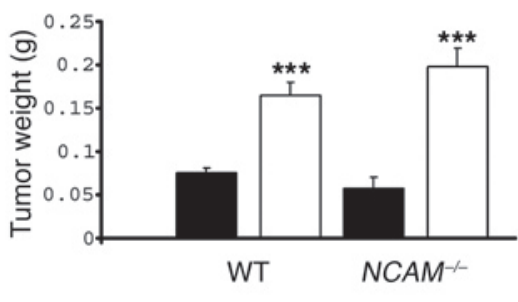

and data not shown), suggesting that expression of NCAM on tumor cells is sufficient for pericyte recruitment to tumor vessels. Stromal PDGF-B retention is required for NCAM's effect on pericyte recruitment. The effects of loss or gain of NCAM in tumor cells are reminiscent of the effects of loss or gain of PDGF-B or PDGFR- $\beta$ in developmental and tumor angiogenesis (21-24). It was therefore important to determine to what extent the NCAM effects were mediated by changes in PDGF-B or PDGFR- $\beta$ expression. Quantitative real-time PCR revealed that both PDGF-B and PDGFR- $\beta$ transcripts were increased in $\mathrm{RT}^{\mathrm{NC} / \mathrm{KO}}$ compared with RT tumors, and decreased in T241 ${ }^{\text {NCAM }}$ compared with T241 ${ }^{\mathrm{eGFP}}$ tumors (Table 1 and Figure 4E). The opposite would have been expected if the effects of NCAM were mediated by changes in PDGF-B/PDGFR- $\beta$ expression levels. Thus, the improved pericyte coverage observed in NCAM-expressing tumors is not caused by increased PDGF-B or PDGFR- $\beta$ expression. Rather, the observed changes in PDGF-B/ $P D G F R-\beta$ expression are instead compatible with a compensatory upregulation of PDGF-B in the pericyte-deficient state, which has been noticed before $(24,25)$.

Recruitment of pericytes to tumor vessels is dependent on the levels and heparin-binding properties of PDGF-B, the latter of which are mediated by a C-terminal stretch of basic amino acids, referred to as the retention motif $(11,22,26)$. To investigate whether NCAM's effect on pericyte recruitment to blood vessels is independent of PDGF-B function, we placed T241 ${ }^{\text {GFP }}$ and T241 NCAM tumors on mice carrying a knock-in mutation in the $P d g f b$ gene, which deletes the ECM-binding retention motif in the PDGF-B protein. This mutation leads to deficient association of pericytes with the abluminal endothelial surface (26). Pericyte recruitment and investment in the tumor vessel walls were severely disturbed in both $\mathrm{T} 241^{\mathrm{eGFP}}$ and T241 ${ }^{\mathrm{NCAM}}$ tumors transplanted on Pdgfb $b^{\text {ret/ret }}$ mice (Figure 4, A-D), indicating that NCAM cannot rescue pericyte deficiency if the host stroma does not provide PDGF-B with the retention motif. Together, these data suggest that the improved pericyte recruitment to T241 NCAM tumors is not mediated by changes in PDGF-B levels but nevertheless depends on proper function of PDGF-B produced by host-derived tumor stroma.

\section{Table 1}

Relative mRNA expression of angiogenesis genes during RT and RTNC/KO tumor progression

\section{Gene}

Neuropilin 1

VEGFR-1 (FIt-1)

VEGFR-2 (FIk-1)

VEGFR-3 (FIk-4)

Angiotensinogen

VEGF-A

VEGF- $B$

VEGF-C

VEGF-D

Angiopoietin-2

PDGF-B

PDGFR- $\alpha$

PDGFR- $\beta$

\section{Real-time PCR ratio, $\mathbf{R T}^{\mathrm{NC} / \mathrm{KO} / \mathrm{RT}}$}

$$
\begin{gathered}
1.1 \\
-1.7^{\mathrm{A}} \\
-1.9^{\mathrm{A}} \\
-3.2^{\mathrm{A}} \\
1.3^{\mathrm{A}} \\
-1.3^{\mathrm{A}} \\
1.1^{\mathrm{A}} \\
-2.5^{\mathrm{A}} \\
-5.0^{\mathrm{A}} \\
1.2^{2} \\
2.3^{\mathrm{A}} \\
-1.4^{\mathrm{A}} \\
1.8^{2}
\end{gathered}
$$

mRNA expression of various angiogenesis genes was quantified by real-time PCR on islets/tumors isolated from 8-week-old RT and RTNC/KO mice. The relative mRNA expression change during $\mathrm{RT}^{\mathrm{NC} / \mathrm{KO}}$ compared with RT tumor progression is given as the RTNC/KO/RT ratio. ${ }^{A}$ Quotas less than 1 were inverted and prefixed with "-" to clarify the fold regulation. 

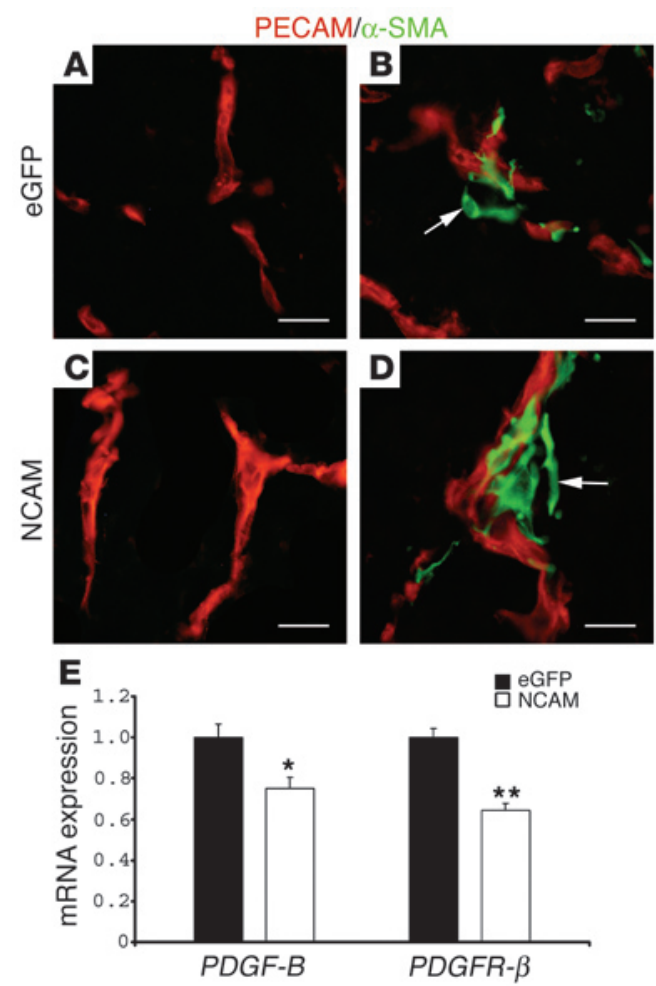

Deficient pericyte-endothelial interaction is sufficient to induce bematogenous and lymphatic tumor cell spreading. Analysis of calponin h1-deficient mice has indicated that the metastatic potential of circulating tumor cells is influenced by the structural integrity of

\section{Figure 4}

NCAM-induced improvement of pericyte recruitment and coverage requires proper PDGF-B production by host-derived tumor stroma. (A-D) Tumor sections of T241 ${ }^{\mathrm{EGFP}}$ ( $\mathbf{A}$ and $\mathbf{B}$ ) and T241NCAM (C and $\left.\mathbf{D}\right)$ cells transplanted on $P d g f b^{r e t / r e t}$ mice double-stained with antibodies against PECAM (red) and $\alpha$-SMA (green). Pericyte recruitment (A and $\mathbf{C}$ ) and association with the endothelium ( $\mathbf{B}$ and $\mathbf{D}$ ) were impaired in the majority of the blood vessels, independent of whether T241 ${ }^{\text {GFFP }}$ or T241 NCAM cells were transplanted. The arrows indicate the detachment of pericytes from the endothelium. Scale bars: $20 \mu \mathrm{m}$. (E) PDGF-B and PDGFR- $\beta$ mRNA expression is downregulated in T241NCAM tumors compared with T241 $1^{\text {GFP }}$ tumors (transplanted on WT mice). Average values \pm SEM are shown. The mean for T241 ${ }^{\mathrm{eGFP}}$ is set to 1 to indicate fold change in T241 ${ }^{\text {NCAM }}$ tumors. ${ }^{*} P<0.05,{ }^{* \star} P<0.01$ (2-tailed Student's $t$ test).

blood vessels (27). Consistent with these observations, we show that the metastatic potential of $\mathrm{RT}^{\mathrm{NC} / \mathrm{KO}}$ tumors correlates with an early-onset disturbance of pericyte-endothelial cell interactions, and vessel leakage. However, hitherto it has been unclear whether pericyte deficiency per se can cause hematogenous spreading of tumor cells from a solid tumor. In order to test this idea, we intercrossed RT mice with Pdgfbet/ret mice (26). The prediction was that if pericyte recruitment to the endothelium prevents tumor cell dissemination, detachment of pericytes during $\beta$ cell tumorigenesis should lead to metastasizing $\beta$ cell tumors in $\mathrm{RTPdg} f b^{\text {ret/ret }}$ mice as it does in $\mathrm{RT}^{\mathrm{NC} / \mathrm{KO}}$ mice. Indeed, when 12 - to 15 -week-old RTPdgfbet/ret mice were analyzed, metastases in distant organs, including the liver, kidney, and intestine, as well as in local lymph nodes were apparent (Figure 5A). Of 7 analyzed RTPdgf $b^{\text {ret/ret }}$ mice, 4 (57\%) developed local lymph node metastasis, of which 3 (43\%) also developed distant metastasis. Metastasizing

\section{Figure 5}

Pericyte detachment causes tumor cell dissemination in distant organs and local lymph nodes. (A) Intercrosses of RT and $P d g f b^{\text {rettret }}$ mice result in $\beta$ tumor cell dissemination in distant organs, including the liver, kidney, and intestine (arrows), and local lymph nodes (arrowheads). (B and C) Immunostainings of sections of RT adenomas and carcinomas and RTPdgfbretret local lymph node and distant organ metastases with $\mathrm{Pdx} 1$ (B) and $\mathrm{E}$-cadherin (C) antibodies. Exocrine and tumor tissues are indicated by "E" and " $T$," respectively. (D-G) Pancreatic tumor sections from RTNC/KO (D and F) and RTPdgfbretret (E and G) mice were stained with $\mathrm{H} \& \mathrm{E}$. Tissue disaggregation and presence of isolated tumor cell clusters inside hemorrhagic lacunae were observed in both genotypes. Isolated cell clusters are indicated by arrows. Scale bars: $1 \mathrm{~cm}(\mathbf{A}), 100 \mu \mathrm{m}(\mathbf{B}-\mathbf{E})$, and $50 \mu \mathrm{m}$ (F and $\mathbf{G})$.
A

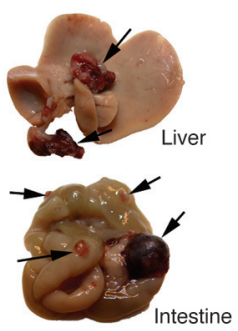

B Adenoma

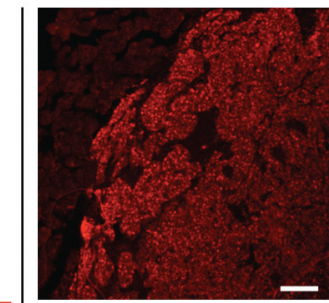

뜸

Intestine

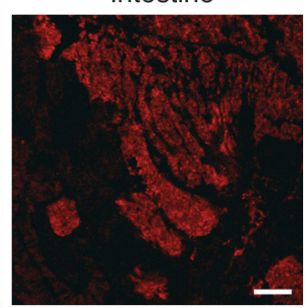

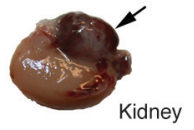

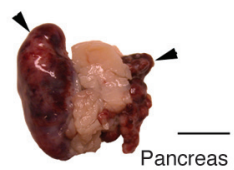

Local lymph node

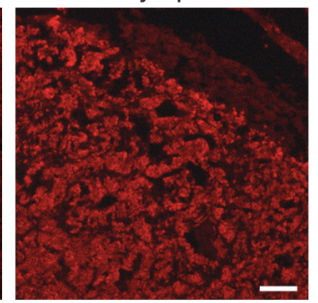

Kidney

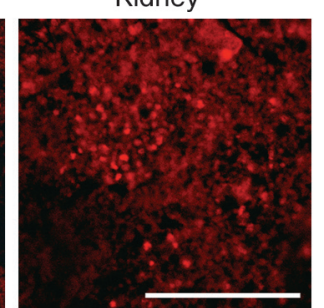

C

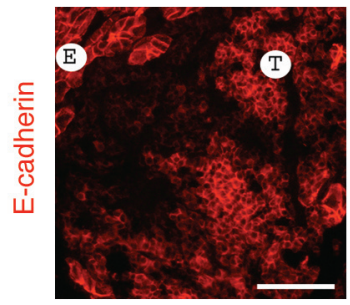

$\mathrm{RT}^{\mathrm{NCAM+1}}$

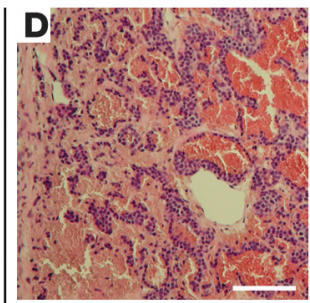

$\mathrm{RT}^{\mathrm{NCAM+1-}}$
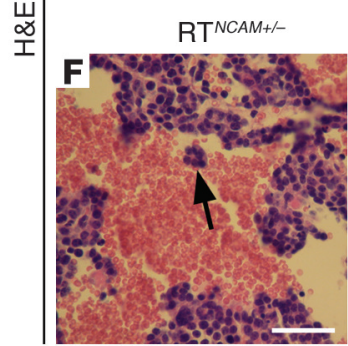

Intestine

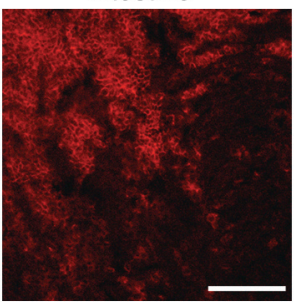

RTPdgf- $b^{\text {ret/ret }}$

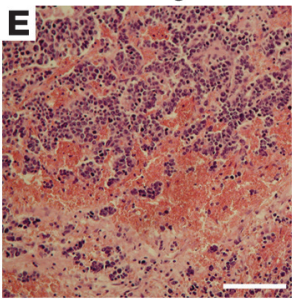

RTPdgf- $b^{\text {ret/ret }}$

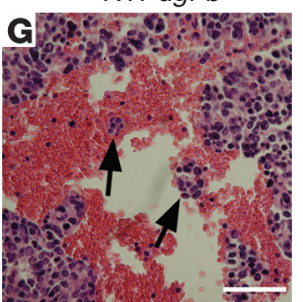



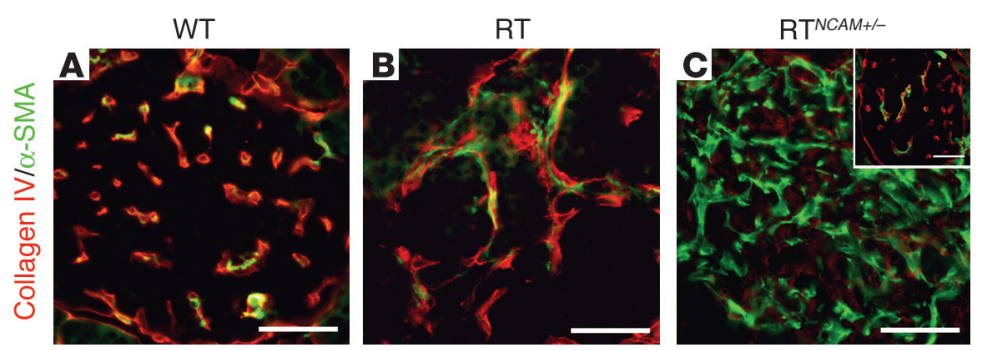

\section{Figure 6}

Severe $\alpha-\mathrm{SMA}^{+}$cellular phenotypes are associated with changes in the expression and distribution of ECM molecules. Pancreas sections of 8-week-old WT (A, D, and $\mathbf{G})$, $\mathrm{RT}$ (B, E, and $\mathbf{H})$, and RT ${ }^{N C A M+l-}(\mathbf{C}, \mathbf{F}$, and I) mice were double-immunostained with antibodies against $\alpha$-SMA (green) and collagen IV (A-C), fibronectin (D-F), and laminin (G-I) (red). In WT islets, $\alpha-\mathrm{SMA}^{+}$cells are closely attached to the blood vessel endothelium. The middle and right columns show angiogenic islets in RT (B, E, and $\mathbf{H})$ and $\mathrm{RT}^{\mathrm{NCAM+/-}}(\mathbf{C}$,
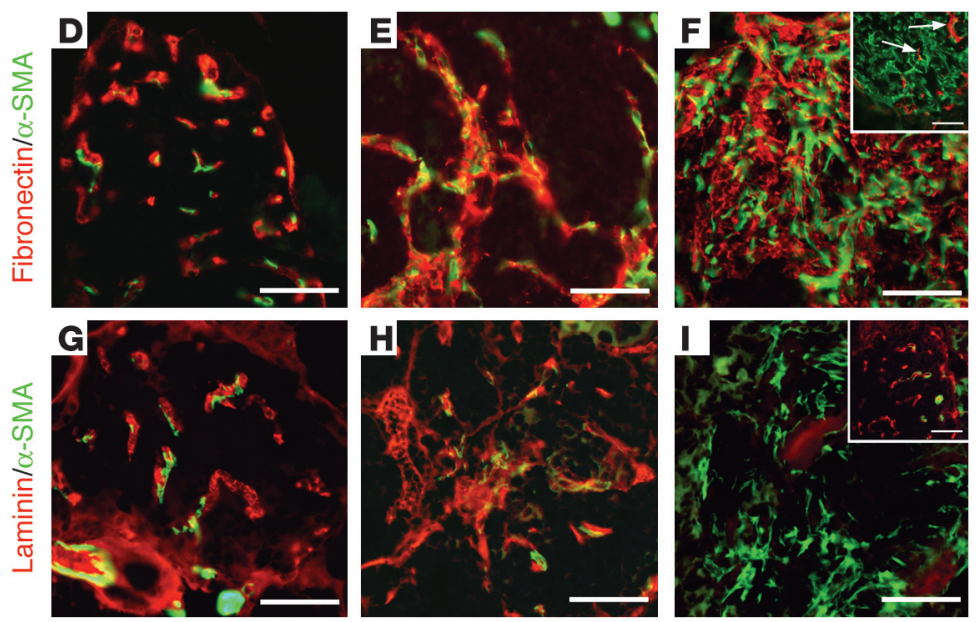
$\mathbf{F}$, and I) mice. Whereas the distribution of the examined ECM molecules in RT islets was commonly observed in $R T^{N C / K O}$ islets, the images of consecutive sections of an

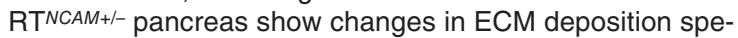
cifically observed in areas with fibroblast-like $\alpha$-SMA ${ }^{+}$cells in RTNC/KO islets, i.e., less deposition of both collagen IV (C) and laminin (I). The insets in $\mathbf{C}$ and I show normal islets from the same sections. The inset in $\mathbf{F}$ shows the same area stained with PECAM (red) antibodies, showing the presence of blood vessels (arrows). In contrast to laminin $\alpha 1$ and collagen IV, fibronectin lost its perivascular distribution and became more widely distributed (F). Scale bars: $50 \mu \mathrm{m}$.

tumor cells were identified by the $\beta$ cell-specific transcription factor Pdx1 (Figure 5B). Undetectable or low levels of insulin expression together with varying levels of E-cadherin expression in the metastases suggested that metastasizing tumor cells underwent dedifferentiation (Figure 5C and data not shown). Metastasizing cells with relatively normal cellular phenotypes expressed basically normal levels of E-cadherin at cell-cell contacts, whereas cells with a malignant phenotype lost E-cadherin expression (Figure 5C). Additionally, histopathological analysis revealed that primary RTPdgf $b^{\text {ret/ret }}$ tumors displayed tissue disaggregation with isolated tumor cell clusters inside hemorrhagic lacunae, as did primary $\mathrm{RT}^{\mathrm{NC} / \mathrm{KO}}$ tumors (Figure 5, D-G). These results suggest that deficient pericyte-endothelial cell-cell interactions are sufficient to induce hematogenous and lymphatic tumor cell spreading.

NCAM regulates perivascular ECM deposition. Recently, we showed that $\mathrm{RT}^{\mathrm{NC} / \mathrm{KO}}$ tumor progression results in a general downregulation of the expression of ECM mRNAs (28). Based on these gene expression changes, we focused our attention on blood vesselassociated ECM molecules. In WT and 8-week-old RT mice, fibronectin, laminin $\alpha 1$, and collagen IV were preferentially associated with endothelial cells within islets (Figure 6, A, B, D, E, G, and H). However, in regions with advanced tumor blood vessel abnormalities, such as detachment of perivascular cells and the presence of fibroblast-like $\alpha-\mathrm{SMA}^{+} \mathrm{NG}^{+}{ }^{+}$desmin $^{+}$cells that were specifically found in $\mathrm{RT}^{\mathrm{NC} / \mathrm{KO}}$ mice, the distribution of all $3 \mathrm{ECM}$ proteins was altered. In these regions, both laminin $\alpha 1$ and collagen IV were dramatically downregulated, whereas fibronectin exhibited a diffuse pattern of distribution (Figure 6, C, F, and I). Importantly, in acinar tissue surrounding the affected angiogenic islets, no change in the distribution of any of the investigated molecules was observed (data not shown), indicating that the changes in perivascular ECM distribution were tumor vessel specific. To further explore whether NCAM regulates the perivascular distribution of ECM molecules, we analyzed T241 $21^{\mathrm{eGFP}}$ and T241 $1^{\mathrm{NCAM}}$ tumors. The prediction was that if NCAM promotes deposition of ECM components around tumor vessels, ectopic expression of NCAM should improve perivascular deposition of ECM molecules. Indeed, we found a general upregulation of fibronectin and laminin $\alpha 1$ both perivascularly and in the tumor tissue of T24 $1^{\mathrm{NCAM}}$ tumors (Figure 7, A-D), whereas collagen IV developed a more pronounced distribution around blood vessels (Figure 7, E and F). Altogether, these findings provide convincing experimental support for an important role of NCAM in maintaining normal deposition of ECM molecules around blood vessels during tumor angiogenesis.

Lymphangiogenesis is not affected during $R T^{N C / K O}$ and RTPdgff ret/ret tumor progression. In both $\mathrm{RT}^{\mathrm{NC} / \mathrm{KO}}$ and $\mathrm{RTPdg} f b^{\text {ret/ret }}$ mice, deficient pericyte interaction with the endothelium was associated with hematogenous and lymphatic tumor cell dissemination. Lymphatic tumor cell dissemination may either arise through direct effects on the lymphatic system, such as increased lymphangiogenesis (29), or secondarily emerge as a consequence of pathological angiogenesis (30). Crnic et al. recently demonstrated that $\mathrm{RT}^{\mathrm{NC} / \mathrm{KO}}$ tumorigenesis is associated with an upregulated expression of the lymphangiogenic factors VEGF-C and VEGF-D and increased lymphangiogenesis (17). A soluble Ig-fusion protein of the ligand-binding domain of the receptor for VEGF-C and -D, VEGFR-3, repressed the function of VEGF-C and -D, resulting in reduced tumor lymphangiogenesis, without, however, affecting lymph node metastasis (17). These results suggest that increased lymphangiogenesis is probably not the sole cause of tumor cell dissemination during $\mathrm{RT}^{\mathrm{NC} / \mathrm{KO}}$ tumor progression. When we examined the abundance and distribution of lymphatic vessels in the vicinity of RT, RT ${ }^{\mathrm{NC} / \mathrm{KO}}$, and RTPdgf $b^{\text {ret/ret }}$ tumors using the specific marker LYVE-1 (31), we confirmed that lymphatic vessels were distributed around, but rarely within, the RT tumors (17). However, we did not observe a signifi- 

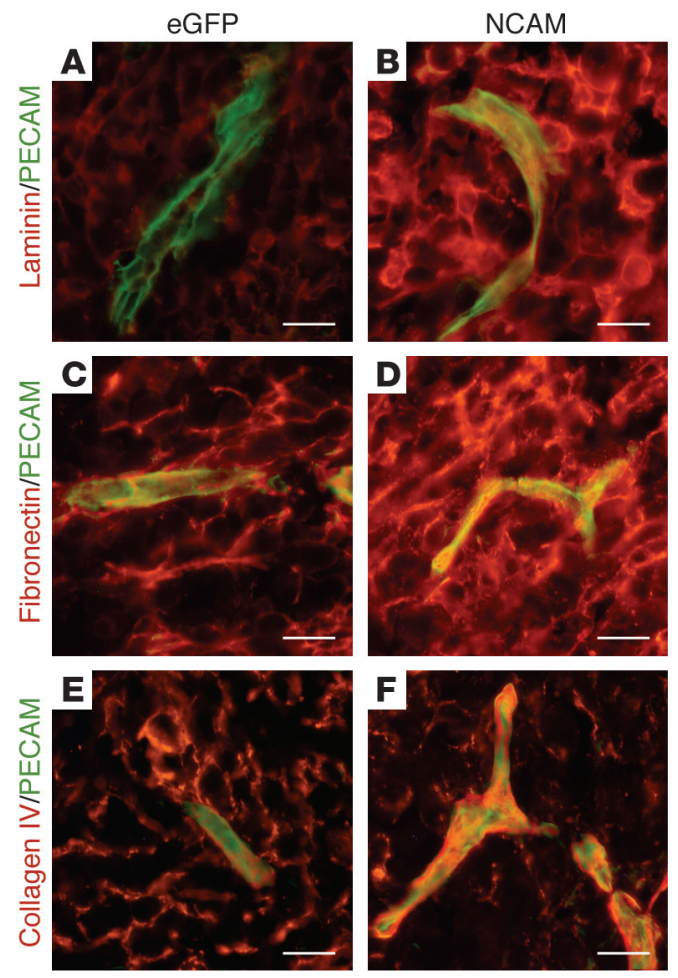

cant difference in the number and distribution of lymphatic vessels among RT, RT ${ }^{\mathrm{NC} / \mathrm{KO}}$, and RTPdgf $b^{\text {ret }}$ /ret tumors (Figure 8, Table 2, and data not shown). In further contrast to Crnic et al., we found that $\mathrm{RT}^{\mathrm{NC} / \mathrm{KO}}$ tumor progression was associated with a downregulated mRNA expression of the lymphangiogenic factors VEGF-C and VEGF-D and their cognate receptor VEGFR-3 compared with RT (2.5-, 5.0-, and 3.2-fold, respectively) (Table 1). Altogether, our findings suggest that lymphangiogenesis was unaffected in the examined tumors.

\section{Discussion}

The metastatic potential of tumors has classically been considered to reflect the cell-autonomous characteristics of a subpopulation of dedifferentiated tumor cells. However, recent studies implicate that the bulk of the primary tumor cells principally carries a similar potential (32), and that the tumor-host microenvironment is relevant to malignancy (33). Also, the metastatic potential of circulating tumor cells has been correlated to the stability of blood vessels (27). However, whether hematogenous spreading of tumor cells from a solid tumor can be caused by pericyte dysfunction has not been addressed.

By analyzing intercrosses of a transgenic insulinoma tumor model, Rip1Tag2 (RT), and a genetic mouse model for primary pericyte dysfunction, $P d g f b^{\text {ret } / r e t}$, we provide genetic evidence that dysfunction of pericytes, reflected by their deficient interaction with the endothelium, is causally involved in hematogenous spreading of RT tumor cells. This is in agreement with a recent study on human colorectal tumor samples, which showed a significant negative correlation between pericyte coverage (SMA staining) and metastasis, as well as poor survival (34). Interestingly, out of a 17 -gene signature recently found to associate with metastatic propensity in various types of human solid tumors (32), 4 of 9 downregulated genes (actin $\gamma 2$, myosin light chain kinase, myosin heavy

\section{Figure 7}

Ectopic expression of NCAM improves deposition of perivascular ECM components in T241 tumors. Sections from T241 $1^{\mathrm{GFP}}$ (A, C, and E) and T241 NCAM (B, D, and F) tumors were stained with antibodies against laminin $\alpha 1$ (red; $\mathbf{A}$ and B), fibronectin (red; C and D), collagen IV (red; $\mathbf{E}$ and $\mathbf{F}$ ), and PECAM (green; $\mathbf{A}-\mathbf{F}$ ). The laminin $\alpha 1$ and fibronectin expression was upregulated both perivascularly and in the tumor tissue in T241 NCAM tumors (B and D) compared with T241 $1^{\text {eGFP }}$ ( $\mathbf{A}$ and $\mathbf{C}$ ). Collagen IV exhibited more distinct distribution to the blood vessels in T241 NCAM tumors (F) compared with T241 ${ }^{\mathrm{eGFP}}(\mathrm{E})$. Scale bars: $20 \mu \mathrm{m}$.

chain 11, and calponin h1) are markers of SMCs. Another study provided a similar negative correlation between the expression of the SMC marker h-caldesmon and metastasis in human melanoma (35). SMC contribution to a solid tumor is mainly in the form of VSMCs/pericytes. Therefore, part of the metastatic gene signature may reflect sparse mural cell coverage of the tumor vessels, leading to destabilization of the vessel wall and increased tumor cell escape into the vasculature. Sparse VSMC/pericyte coverage is indeed commonly found in both human and experimental solid tumors $(11,16,36)$. Alternatively, downregulation of the abovementioned SMC markers in the tumor vasculature may reflect an altered state of differentiation of the VSMC, which may likewise exert a destabilizing effect on the tumor vasculature. While VSMC/ pericyte deficiency within the tumor vessels may facilitate tumor cell spreading into the circulation, similar mechanisms may affect extravasations of circulating tumor cells. The increased metastasis of intravenously injected melanoma cells in calponin h1-deficient mice supports this notion (27).

Our observations suggest that NCAM plays an important role in stabilizing the vessel wall by promoting pericyte-endothelial cell interactions. Using both loss-of-function and gain-of-function approaches in 2 independent tumor models, we show that NCAM regulates tumor-associated pathological angiogenesis. Whereas NCAM deficiency during RT tumor progression resulted in premature detachment of pericytes, ectopic expression of NCAM in T241 fibrosarcomas resulted in enhanced pericyte recruitment and coverage during tumor progression. Interestingly, expression of NCAM also resulted in a 2-fold increase in tumor growth, which appeared to correlate with an increased tumor vessel density. Transplantation of T $241^{\mathrm{NCAM}}$ cells on NCAM-deficient mice demonstrated that NCAM's effects on the vasculature did not require homophilic NCAM tumor-stroma interactions. Moreover,
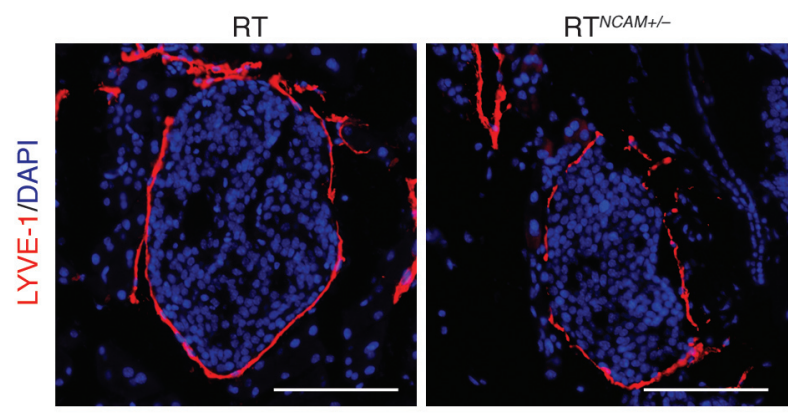

\section{Figure 8}

Lymphangiogenesis is unaffected during RTNC/KO tumor progression. Immunostainings of sections of RT (left) and RTNCAM+/- (right) islets with LYVE-1 antibodies (red). Nuclei are indicated by DAPI (blue). Scale bars: $100 \mu \mathrm{m}$. 


\section{Table 2}

Lymphangiogenesis during $\mathrm{RT}$ and $\mathrm{RT} \mathrm{NC}^{\mathrm{N} / \mathrm{KO}}$ tumor progression

\begin{tabular}{|c|c|c|c|c|c|c|}
\hline \multirow[t]{2}{*}{ Age } & \multirow[t]{2}{*}{ Genotype } & \multirow[t]{2}{*}{ Mice } & \multirow[t]{2}{*}{$\begin{array}{l}\text { Islets/ } \\
\text { Tumors }\end{array}$} & \multicolumn{3}{|c|}{$\begin{array}{l}\text { Lymphatic vessel } \\
\text { density }^{A}(\%)\end{array}$} \\
\hline & & & & 0 & $<50$ & $>50$ \\
\hline 8 Weeks & RT & 9 & 261 & 47.9 & 51.3 & 0.8 \\
\hline 8 Weeks & $\mathrm{RT} N \mathrm{NC} / \mathrm{KO}$ & 6 & 204 & 45.1 & 54.4 & 0.5 \\
\hline 14 Weeks & RT & 8 & 225 & 36.0 & 60.4 & 3.6 \\
\hline 14 Weeks & $\mathrm{RTNC/KO}$ & 7 & 237 & 43.0 & 53.2 & 3.8 \\
\hline
\end{tabular}

ALymphatic vessels were identified with LYVE-1 immunostaining. Lymphatic vessel density was determined by assessment of the percentage of the islet/tumor perimeter covered by lymphatic vessels. There is no significant difference between RT and $\mathrm{RT}^{\mathrm{NC} / \mathrm{KO}}$ ( $\chi^{2}$ test).

transplantations of T2 $41^{\text {NCAM }}$ cells on $P d g f b^{\text {ret/ret }}$ mice showed that NCAM's positive effect on VSMC/pericyte recruitment depends on proper PDGF-B production by the host.

How could NCAM expression in tumor cells affect integration of pericytes in the tumor vessel wall? Based on current knowledge of the molecular mechanisms for pericyte recruitment and coverage, this could involve changes in the expression/distribution of PDGF-B and/or PDGFR- $\beta$, or changes in the perivascular composition of ECM molecules (8). The observed changes in PDGF-B and PDGFR- $\beta$ expression in the NCAM-deficient and NCAM-expressing tumors were, however, opposite to what could be expected for a system mediating the NCAM effects, and instead consistent with a compensatory change.

The experiments involving T241 transplantation to Pdgfb $\mathrm{b}^{\mathrm{ret} / \mathrm{ret}}$ mice nevertheless suggested that the retention motif in the host PDGF-B is required for tumor cell NCAM to be able to increase pericyte coverage. The question remains: how can these 2 molecules expressed in 2 different cell types act together on the third cell type, the pericyte? Two possibilities may be considered. First, NCAM itself could directly bind and enhance PDGF-B function in a retention motif-dependent manner. Although untested, this is a possibility, as NCAM has been found to be heavily sulfated at an unusual poly-sialic acid group linked to one of the extracellular Ig domains (37). This sialyl-type sulfated N-linked glycan should, in theory, provide excellent retention of growth factors that contain a basic amino acid retention motif. Second, NCAM could affect PDGF function indirectly by regulating expression and deposition of ECM molecules that are responsible for PDGF-B retention. NCAM-deficient tumors show changes in the expression of a number of ECM molecules implicated in vascular basement membrane integrity (28). One of the downregulated ECM components is the small leucine-rich-repeat proteoglycan biglycan, which is involved in fibrillar assembly of collagen in basement membranes. Biglycan is sulfated and, as such, a hypothetical PDGF-B retention motif-binding molecule. A recent study indicates that biglycan overexpression can increase VSMC migration and proliferation (38).

How are the changes in ECM components related to tumor cell dissemination? NCAM ablation during $\beta$ tumor cell progression results in an overall diminished mRNA and protein expression of several other ECM molecules (28). We examined the distribution of ECM molecules around $\mathrm{RT}^{\mathrm{NC} / \mathrm{KO}}$ tumor blood vessels and discovered defective perivascular deposition of fibronectin, and of the basal lamina components laminin $\alpha 1$ and collagen IV, in areas with severely disturbed endothelial-mural cell-cell interactions.
Conversely, we found that ectopic expression of NCAM in T241 fibrosarcoma cells resulted in increased expression and perivascular distribution of the same ECM molecules. Altogether, these findings suggest that NCAM plays an important role in maintaining normal deposition of ECM molecules around blood vessels during pathological angiogenesis. The formation of a perivascular matrix, including the basement membrane, is important for stabilization of the vessel wall (8). Thus, NCAM may limit tumor cell dissemination by stabilizing tumor vessels through the synthesis of perivascular ECM components, which in turn facilitates pericyte integration in the vessel wall.

Whereas the specific pericyte phenotype in Pdgf $b^{\text {ret } / \text { ret }}$ mice (26) indicates that the lymphatic spreading of tumor cells in RTPdgf $b^{\text {ret }} /$ ret mice is secondarily caused by the blood vessel-related changes, it is unclear whether the lymphatic tumor cell dissemination in $\mathrm{RT}^{\mathrm{NC} / \mathrm{KO}}$ mice also is secondary to the blood vessel phenotype or involves direct effects on the lymphatic system. Based on the finding that $\mathrm{RT}^{\mathrm{NC} / \mathrm{KO}}$ tumor progression is associated with increased expression of the lymphangiogenic factors VEGF-C and VEGF-D and enhanced lymphangiogenesis, it was recently suggested that increased lymphangiogenesis may underlie NCAM-deficient $\beta$ tumor cell metastasis (17). In contrast, we found no evidence for increased lymphangiogenesis during $\mathrm{RT}^{\mathrm{NC} / \mathrm{KO}}$ tumorigenesis. Also, we found that VEGF-C, VEGF-D, and their cognate receptor VEGFR-3 were all downregulated in their expression during $\mathrm{RT}^{\mathrm{NC} / \mathrm{KO}}$ tumor progression (Table 1). Since inhibition of lymphangiogenesis during NCAM-deficient tumor progression did not correlate with inhibition of lymph node metastasis (17), alternative explanations for the increase in this type of metastasis in RT ${ }^{\mathrm{NC} / \mathrm{KO}}$ animals need to be considered. Possibly, lymph node metastasis in $\mathrm{RTPdg} f b^{\text {ret } / \text { ret }}$ and $\mathrm{RT} \mathrm{NC}^{\mathrm{NCO}}$ animals may arise as a secondary consequence of the deficient blood vessel structure and increased leakage in RTPdgff ret/ret and RT ${ }^{\mathrm{NC} / \mathrm{KO}}$ tumors. For example, it appears plausible that changes in the composition of the interstitial fluid that occur as a consequence of blood vessel dysfunction may promote tumor cell escape into the lymphatics.

In summary, our study suggests that tumor cell-expressed NCAM molecules, through their effects on matrix deposition and pericyte recruitment, may stabilize and normalize tumor vessel morphology and function and thereby limit tumor metastasis. Normalization of tumor vasculature is an emerging concept in antiangiogenic therapy, which may have the combined beneficial effects of increasing drug delivery and the impact of radiation and improving host immune responses - effects that are not necessarily counterbalanced by an accelerated tumor growth as a result of improved oxygenation and nutrition (reviewed in refs. 39, 40). Our current study suggests the possibility of additional beneficial effects of tumor vessel normalization, namely decreased blood-borne metastatic dissemination of tumor cells.

\section{Methods}

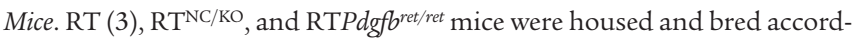
ing to Swedish animal research regulations. Approval for the performed animal experiments was obtained from the animal ethical committees at Gothenburg and Lund Universities. NCAM-deficient mice (C57BL/ 6 background) (41) were crossed with RT mice (C57BL/6 background) to generate $\mathrm{RT}^{\mathrm{NC} / \mathrm{KO}}$ and RT littermates. Pdgffret/ret mice (26) were crossed with RT mice to obtain RTPdgffret/ret mice. Glucose (5\% weight/volume) was fed to all tumor-bearing mice when they reached 6 weeks of age to compensate for the hypoglycemia induced by the insulinomas. 
Immunoreagents. Antibodies used for immunostainings include rat antiPECAM (1:200; BD), FITC- and Cy3-conjugated anti- $\alpha$-SMA (1:100; SigmaAldrich), guinea pig anti-insulin (1:750; Linco Research Inc.), rabbit antiKi67 (1:200; Novocastra Laboratories Ltd.), rat anti-E-cadherin (1:40 [ref. 42]), rabbit anti-collagen IV (1:250; Biogenesis Ltd.), rabbit anti-fibronectin (1:200; Dako), mouse monoclonal anti-laminin $\alpha 1$ (generous gift from $\mathrm{P}$. Ekblom, Biomedical Center, Lund University), rabbit anti-LYVE (generous gift from D. Jackson, Institute of Molecular Medicine, John Radcliffe Hospital, Oxford, United Kingdom, 1:200; and Upstate, 1:500), rabbit anti-Pdx1 (generous gift from C. Wright, Vanderbilt University School of Medicine, Nashville, Tennessee, USA), biotin-conjugated donkey anti-rabbit (1:1,000; Jackson ImmunoResearch Laboratories Inc.), biotin-conjugated donkey anti-rat (1:750; Jackson ImmunoResearch Laboratories Inc.), Cy3-conjugated goat anti-rat (1:100; Jackson ImmunoResearch Laboratories Inc.), Cy3-conjugated goat anti-rabbit (1:100; Jackson ImmunoResearch Laboratories Inc.), Cy3-conjugated streptavidin (1:1,500; Jackson ImmunoResearch Laboratories Inc.), FITC-conjugated donkey anti-rat (1:100; Jackson ImmunoResearch Laboratories Inc.), and streptavidin-Alexa 633 (1:100; Invitrogen Corp.). DAPI (1:2,000; Invitrogen Corp.) was used for labeling of nuclei.

Immunohistochemistry. Mice were sacrificed by cervical dislocation, and the pancreas was fixed in $4 \%$ paraformaldehyde and processed according to standard procedure for frozen and paraffin embedding. Ten- and fiftymicrometer cryosections and $5-\mu \mathrm{m}$ paraffin sections were stained with different primary and secondary antibodies. Samples were analyzed with a Zeiss conventional light and fluorescence microscope. Thicker sections $(50 \mu \mathrm{m})$ were scanned with a TCS-NT laser-scanning confocal microscope (Leica Microsystems Inc.).

FITC-dextran perfusion and $\alpha$-SMA morphometrics. Eight-week-old mice were deeply anesthetized with i.p. injection of Avertin (2.5\%, $10 \mu \mathrm{l} / \mathrm{g}$ body weight), the chest was opened, and the vasculature was perfused through the heart with $5 \mathrm{ml}$ FITC-conjugated dextran (average molecular weight $2 \times 10^{6}$ ) (Sigma-Aldrich) at constant pressure. The pancreas was dissected out, fixed, and sectioned $(50 \mu \mathrm{m})$. To localize the islets and to be able to distinguish between intravascular and extravascular dextran, sections were stained with antibodies against insulin and PECAM. Alternatively, the sections were stained with antibodies against insulin and Cy3-conjugated $\alpha$-SMA. The specimens were scanned with a Leica TCS-NT laser-scanning confocal microscope as described above. As controls, brain and skin tissues were processed and stained with an antibody against PECAM to evaluate vascular leakage in other tissues. By visual inspection of confocal images of islets $(n=293)$, the degree of leakage was graded into 4 classes (0-3) according to the size of the intra-islet leakage area, i.e., the fraction of the islet area that was covered with FITC-dextran. The definition of each grade is as follows: grade 0 , no leakage; grade 1 , approximately $0-5 \%$; grade 2 , approximately $5-30 \%$; grade 3 , more than $30 \%$. The evaluation was performed blind and repeated twice. For $\alpha$-SMA morphometrics, islets were classified into 2 groups according to $\alpha$-SMA cellular phenotypes. Islets were considered normal if all blood vessels had a normal coverage of $\alpha-\mathrm{SMA}^{+}$cells, and disturbed if 1 or more vessels either had sheetlike structures of $\alpha-\mathrm{SMA}^{+}$cells or showed detachment of $\alpha$-SMA ${ }^{+}$cells from the endothelium.

Retinal examination. Retinas from XlacZ4/NCAM+/+ and XlacZ4/NCAM ${ }^{+/-}$ mice at postnatal day 5 and 3 weeks of age were prepared and stained with $\mathrm{X}$-gal and isolectin as previously described (43).

Vessel density ( $R T$ and $\left.R T^{\mathrm{NC} / K O}\right)$. Ten-micrometer cryosections were stained against PECAM (CD31), and pictures of the pancreatic islets of Langerhans were taken at $\times 400$ magnification under a fluorescent microscope. Pictures of 112 islets from $3 \mathrm{RT}$ mice and 95 islets from $3 \mathrm{RT}^{\mathrm{NC} / \mathrm{KO}}$ mice were taken. Depending on the size of the islet, 1-6 pictures were taken of each islet. In pictures where the islet did not fill up the whole picture, the islet area was measured with ImageJ computer software (NIH, Bethesda, Maryland,
USA). The number of profiles was calculated per area of $0.004 \mathrm{~mm}^{2}=1$ area unit. For statistics, the Student's $t$ test was used.

T241 fibrosarcoma cell culture, transfection, and transplantation. T241 fibrosarcoma cells were propagated in DMEM with $10 \%$ FCS and standard supplements. The cells were transfected with vector containing murine NCAM-140 cDNA or eGFP driven by the CMV promoter (pRc/CMV; Invitrogen Corp.) using Lipofectamine Reagent according to the manufacturer's instructions (Invitrogen Corp.). For selection, neomycin resistance was used. The NCAM-transfected cells were also selected in a second round using rabbit anti-NCAM antibody and Dynabeads coated with sheep anti-rabbit antibody (Dynal Biotech). Expression of eGFP and NCAM was confirmed by eGFP detection and immunofluorescence stainings. Proliferation studies were performed by seeding of $1 \times 10^{4} \mathrm{cells} / \mathrm{cm}^{2}$ and then counting of the cells after 1,3 , and 6 days. For the tumors, $1 \times 10^{6}$ cells in $100 \mu$ l PBS were injected s.c. in 2 places on the backs of C57BL/ 6 or NCAM-deficient mice. After 9 days, the tumors were dissected, their width, height, and weight were measured, and they were further processed for histological analysis. The tumor growth curve experiment was performed by injection of $1 \times 10^{6}$ cells in $100 \mu \mathrm{l}$ PBS s.c. in 2 places on the backs of C57BL/6 mice. Tumors were dissected and weighed after 5, 7, 9, and 11 days.

Vessel density (T241 fibrosarcoma). Pictures of a $0.0361-\mathrm{mm}^{2}$ area (here defined as an area unit) were taken at $\times 400$ magnification. Pictures from a green and a red filter were taken separately and merged in Photoshop. Four tumors from $\mathrm{T} 241^{\mathrm{eGFP}}$ mice and four from T241 ${ }^{\mathrm{NCAM}}$ mice were used for the analyses. Nine random pictures were taken on each section, and 5 sections per tumor were analyzed. For the vessel density, the number of vessel profiles and $\alpha-\mathrm{SMA}^{+}$ profiles was calculated. For the pericyte coverage analysis, the $\mathrm{PECAM}^{+}$and $\alpha$-SMA ${ }^{+}$area was measured using Openlab 2.0.7 (Improvision). Pericyte coverage was measured by calculation of the overlap of $\alpha$-SMA and PECAM. Pericyte recruitment was calculated by relation of the $\alpha$-SMA-stained area to the anti-PECAM-stained area. Pericyte integration was calculated by division of the $\alpha$-SMA ${ }^{+} \mathrm{PECAM}^{+}$area by the $\alpha$-SMA ${ }^{+} \mathrm{PECAM}^{-}$area. Mean values \pm SEM are shown. Differences were analyzed by Student's $t$ test.

Real-time PCR. The material used was RNA isolated from pancreatic islets of 8-week-old RT and RT ${ }^{\mathrm{NC} / \mathrm{KO}}$ mice. Total RNA was prepared using the RNeasy Mini Kit (QIAGEN) with the RNase-Free DNase (QIAGEN) treatment according to the manufacturer's instructions. RNA was pooled from 6 $\mathrm{RT}$ and $7 \mathrm{RT} \mathrm{TC}^{\mathrm{NCO}}$ mice, respectively. cDNA synthesis and 2 rounds of RNA amplification starting from $2 \mu \mathrm{g}$ total RNA were performed as previously described (44), except that no linear acrylamide was used in the first round. The primers used were: VEGF-D: forward, 5 -AGCCAGGAGAACCCTTGATT-3'; reverse, 5'-AGTGGGCAACAGTGACAGCA-3'; Flk-4: forward, 5'CCACACAGAACTCTCCAGCA-3'; reverse, $5^{\prime}$-GAGCCACTCGACACTGATGA-3'; angiotensinogen: forward, $5^{\prime}$-CTGACCCAGTTCTTGCCACT-3'; reverse, 5'-CACCGAGATGCTGTTGTCC-3'; PDGF-B: forward, 5'-GAGCACAGACTGGAGGAAC-3'; reverse, 5'-GTAGGGGAAGTGGAAAGAGG3'; PDGFR- $\alpha$ : forward, 5'-TCCAGTAGTTCCACCTTCATC-3'; reverse, $5^{\prime}$-TTTCTCTCTCCACATCACCC-3'; PDGFR- $\beta$ : forward, $5^{\prime}$-CCAGCAGGTAGATGAGGAG-3'; reverse, 5'-CAGGAGATGGTGGAGGAAG-3'. Primer sequences for VEGF-A, VEGF-B, VEGF-C, neuropilin, Flt-1, Flk-1, $\beta$-tubulin, and angiopoietin- 2 are described elsewhere (28). The data were normalized against $\beta$-tubulin. Real-time PCR measurements were carried out as previously described $(45,46)$.

Statistics. Data were analyzed using 2-tailed Student's $t$ test. $P$ values less than 0.05 were considered statistically significant.

\section{Acknowledgments}

We thank Gunilla Petersson, Sara Tilander, Maria Simonen, and Ingela Berglund-Dahl for technical assistance. We thank C. Wright, P. Ekblom, and D. Jackson for providing antibodies. This work was 
supported by the Swedish Cancer Foundation, the Association for International Cancer Research (United Kingdom), and the Lundberg, Wallenberg, and Söderberg Foundations. H. Gerhardt is supported by Cancer Research UK.

Received for publication May 20, 2005, and accepted in revised form December 6, 2005.

1. Ellis, L.M., and Fidler, I.J. 1996. Angiogenesis and metastasis. Eur. J. Cancer. 32A:2451-2460.

2. Thiery, J.P. 2002. Epithelial-mesenchymal transitions in tumour progression. Nat. Rev. Cancer. 2:442-454.

3. Hanahan, D. 1985. Heritable formation of pancreatic beta-cell tumours in transgenic mice expressing recombinant insulin/simian virus 40 oncogenes. Nature. 315:115-122.

4. Perl, A.K., et al. 1999. Reduced expression of neural cell adhesion molecule induces metastatic dissemination of pancreatic beta tumor cells. Nat. Med. 5:286-291.

5. Roesler, J., Srivatsan, E., Moatamed, F., Peters, J., and Livingston, E.H. 1997. Tumor suppressor activity of neural cell adhesion molecule in colon carcinoma. Am. J. Surg. 174:251-257.

6. Tezel, E., Kawase, Y., Takeda, S., Oshima, K., and Nakao, A. 2001. Expression of neural cell adhesion molecule in pancreatic cancer. Pancreas. 22:122-125.

7. Fogar, P., et al. 1997. Neural cell adhesion molecule (N-CAM) in gastrointestinal neoplasias. Anticancer Res. 17:1227-1230.

8. Allt, G., and Lawrenson, J.G. 2001. Pericytes: cell biology and pathology. Cells Tissues Organs. 169:1-11.

9. Armulik, A., Abramsson, A., and Betsholtz, C. 2005. Endothelial/pericyte interactions. Circ. Res. 97:512-523.

10. Baluk, P., Hashizume, H., and McDonald, D.M. 2005. Cellular abnormalities of blood vessels as targets in cancer. Curr. Opin. Genet. Dev. 15:102-111.

11. Abramsson, A., et al. 2002. Analysis of mural cell recruitment to tumor vessels. Circulation. 105:112-117.

12. Bergers, G., Song, S., Meyer-Morse, N., Bergsland, E., and Hanahan, D. 2003. Benefits of targeting both pericytes and endothelial cells in the tumor vasculature with kinase inhibitors. J. Clin. Invest. 111:1287-1295. doi:10.1172/JCI200317929.

13. Betsholtz, C., Karlsson, L., and Lindahl, P. 2001. Developmental roles of platelet-derived growth factors. Bioessays. 23:494-507.

14. Lindahl, P., et al. 1999. Role of platelet-derived growth factors in angiogenesis and alveogenesis. Curr. Top. Pathol. 93:27-33.

15. Lindahl, P., Johansson, B.R., Leveen, P., and Betsholtz, C. 1997. Pericyte loss and microaneurysm formation in PDGF-B-deficient mice. Science. 277:242-245.

16. Hashizume, H., et al. 2000. Openings between defective endothelial cells explain tumor vessel leakiness. Am. J. Pathol. 156:1363-1380.

17. Crnic, I., et al. 2004. Loss of neural cell adhesion

Address correspondence to: Henrik Semb, Stem Cell Center, Lund University, BMC, B10, Klinikgatan 26, SE-22184 Lund, Sweden. Phone: 46462223159; Fax: 46462223600; E-mail: Henrik.Semb@med.lu.se.

Xiaojie Xian and Joakim Håkansson contributed equally to this work.

molecule induces tumor metastasis by up-regulating lymphangiogenesis. Cancer Res. 64:8630-8638.

18. Morikawa, S., et al. 2002. Abnormalities in pericytes on blood vessels and endothelial sprouts in tumors. Am. J. Pathol. 160:985-1000.

19. Rucker, H.K., Wynder, H.J., and Thomas, W.E. 2000. Cellular mechanisms of CNS pericytes. Brain Res. Bull. 51:363-369.

20. Tidhar, A., et al. 2001. A novel transgenic marker for migrating limb muscle precursors and for vascular smooth muscle cells. Dev. Dyn. 220:60-73.

21. Tallquist, M.D., French, W.J., and Soriano, P. 2003. Additive effects of PDGF receptor beta signaling pathways in vascular smooth muscle cell development. PLoS Biol. 1:E52.

22. Abramsson, A., Lindblom, P., and Betsholtz, C. 2003. Endothelial and nonendothelial sources of PDGF-B regulate pericyte recruitment and influence vascular pattern formation in tumors. J. Clin. Invest. 112:1142-1151. doi:10.1172/JCI200318549.

23. Furuhashi, M., et al. 2004. Platelet-derived growth factor production by B16 melanoma cells leads to increased pericyte abundance in tumors and an associated increase in tumor growth rate. Cancer Res. 64:2725-2733.

24. Hellstrom, M., et al. 2001. Lack of pericytes leads to endothelial hyperplasia and abnormal vascular morphogenesis. J. Cell Biol. 153:543-553.

25. Hellstrom, M., Kalen, M., Lindahl, P., Abramsson, A., and Betsholtz, C. 1999. Role of PDGF-B and PDGFR-beta in recruitment of vascular smooth muscle cells and pericytes during embryonic blood vessel formation in the mouse. Development. 126:3047-3055.

26. Lindblom, P., et al. 2003. Endothelial PDGF-B retention is required for proper investment of pericytes in the microvessel wall. Genes Dev. 17:1835-1840.

27. Taniguchi, S., et al. 2001. Structural fragility of blood vessels and peritoneum in calponin h1-deficient mice, resulting in an increase in hematogenous metastasis and peritoneal dissemination of malignant tumor cells. Cancer Res. 61:7627-7634.

28. Håkansson, J., et al. 2005. N-CAM-deficient beta cell tumorigenesis results in diminished ECM molecule expression and tumor cell-matrix adhesion. Tumor Biol. 26:103-112.

29. Jussila, L., and Alitalo, K. 2002. Vascular growth factors and lymphangiogenesis. Physiol. Rev. 82:673-700.

30. Pepper, M.S., and Skobe, M. 2003. Lymphatic endothelium: morphological, molecular and functional properties. J. Cell Biol. 163:209-213.

31. Mandriota, S.J., et al. 2001. Vascular endothelial growth factor-C-mediated lymphangiogenesis promotes tumour metastasis. EMBO J. 20:672-682.
32. Ramaswamy, S., Ross, K.N., Lander, E.S., and Golub, T.R. 2003. A molecular signature of metastasis in primary solid tumors. Nat. Genet. 33:49-54.

33. Liotta, L.A., and Kohn, E.C. 2001. The microenvironment of the tumour-host interface. Nature. 411:375-379.

34. Yonenaga, Y., et al. 2005. Absence of smooth muscle actin-positive pericyte coverage of tumor vessels correlates with hematogenous metastasis and prognosis of colorectal cancer patients. Oncology. 69:159-166.

35. Koganehira, Y., et al. 2003. Reduced expression of actin-binding proteins, h-caldesmon and calponin $\mathrm{h} 1$, in the vascular smooth muscle inside melanoma lesions: an adverse prognostic factor for malignant melanoma. Br. J. Dermatol. 148:971-980.

36. Schlingemann, R.O., et al. 1991. Differential expression of markers for endothelial cells, pericytes, and basal lamina in the microvasculature of tumors and granulation tissue. Am. J. Pathol. 138:1335-1347.

37. von Der Ohe, M., et al. 2002. Localization and characterization of polysialic acid-containing $\mathrm{N}$-linked glycans from bovine NCAM. Glycobiology. 12:47-63.

38. Shimizu-Hirota, R., et al. 2004. Extracellular matrix glycoprotein biglycan enhances vascular smooth muscle cell proliferation and migration. Circ. Res. 94:1067-1074.

39. Jain, R.K. 2003. Molecular regulation of vessel maturation. Nat. Med. 9:685-693.

40. Jain, R.K. 2005. Normalization of tumor vasculature: an emerging concept in antiangiogenic therapy. Science. 307:58-62.

41. Cremer, H., et al. 1994. Inactivation of the N-CAM gene in mice results in size reduction of the olfactory bulb and deficits in spatial learning. Nature. 367:455-459.

42. Shirayoshi, Y., Nose, A., Iwasaki, K., and Takeichi, M. 1986. N-linked oligosaccharides are not involved in the function of a cell-cell binding glycoprotein E-cadherin. Cell Struct. Funct. 11:245-252.

43. Enge, M., et al. 2002. Endothelium-specific plateletderived growth factor-B ablation mimics diabetic retinopathy. $E M B O J .21: 4307-4316$.

44. Scheidl, S.J., et al. 2002. mRNA expression profiling of laser microbeam microdissected cells from slender embryonic structures. Am. J. Pathol. 160:801-813.

45. Stahlberg, A., Hakansson, J., Xian, X., Semb, H., and Kubista, M. 2004. Properties of the reverse transcription reaction in mRNA quantification. Clin. Chem. 50:509-515.

46. Stahlberg, A., Zoric, N., Aman, P., and Kubista, M. 2005. Quantitative real-time PCR for cancer detection: the lymphoma case. Expert Rev. Mol. Diagn. 5:221-230. 